\title{
Finite size effects in classical string solutions of the Schrödinger geometry
}

\author{
Dimitrios Zoakos \\ Department of Physics, National and Kapodistrian University of Athens, \\ Athens 15784, Greece \\ Hellenic American University, \\ 436 Amherst st, Nashua, NH 03063, U.S.A. \\ E-mail: zoakos@gmail.com
}

Abstract: We study finite size corrections to the semiclassical string solutions of the Schrödinger spacetime. We compute the leading order exponential corrections to the infinite size dispersion relation of the single spin giant magnon and of the single spin single spike solutions. The solutions live in a $S^{3}$ subspace of the five-sphere and extent in the Schrödinger part of the metric. In the limit of zero deformation the finite size dispersion relations flow to the undeformed $\mathrm{AdS}_{5} \times S^{5}$ counterparts and in the infinite size limit the correction term vanishes and the known infinite size dispersion relations are obtained.

KeYwords: AdS-CFT Correspondence, Duality in Gauge Field Theories, Integrable Field Theories, Space-Time Symmetries

ARXiv EPrint: 2006.02285 


\section{Contents}

1 Introduction 1

2 Classical string solutions on $\mathrm{Sch}_{5} \times S^{3} \quad 2$

2.1 Ansatz and equations of motion 3

2.2 Boundary conditions 4

3 Single spin giant magnon $\quad 5$

$\begin{array}{lll}4 & \text { Single spin single spike } & 7\end{array}$

5 Conclusions $\quad 8$

A Useful expressions from the analysis of the classical string solutions $\quad 9$

B Elliptic integrals and $\epsilon$ expansion coefficients of the parameter $v \quad 9$

\section{Introduction}

The powerful tool of integrability (for a review see [1]) is behind all the recent advances in understanding the celebrated gauge-gravity correspondence [2] (for a set of pedagogical introductions see $[3,4]$ ) in the planar limit. On the gauge theory side, using Bethe ansatz techniques [5], the matrix of the anomalous dimensions can be diagonalized through the mapping to the Hamiltonian of an integrable spin chain. The magnons are the fundamental single particle excitations that propagate on the BMN vacuum and integrability implies that every scattering process factorizes in a scattering between two magnons. The role of integrability is pivotal not only in determining the spectrum of $\mathcal{N}=4 \mathrm{SYM}$ at any value of the coupling but also in the computation of higher point correlation functions (see e.g. $[6-11])$.

The strong coupling dual of the magnons are semi-classical string solutions on $\mathrm{AdS}_{5} \times$ $S^{5}$, the so-called giant magnons [12-15]. They are open strings moving in a subspace of the five-sphere with finite angular extent. Another class of classical solutions that we will focus our attention in the current paper is the single spikes [16, 17]. They have finite angular amplitude and wind infinitely many times around an angular direction.

Over the years there is a lot of attention on integrable deformations of the original AdS/CFT construction. Recent activity is coming from the null dipole deformation of the $\mathcal{N}=4 \mathrm{SYM}$ and more precisely on the integrability issues that arise, as they were discussed [18]. There, a test of the Schrödinger holography is provided by matching the anomalous dimensions of long gauge theory operators with the prediction at strong coupling of certain BMN-like strings (see also [19]). 
In [20] semi-classical string solutions living in the Schrödinger spacetime were constructed. They are the counterparts of the giant magnon and the single spike solutions of the undeformed $\mathrm{AdS}_{5} \times S^{5}$, since in the limit of zero deformation they flow to the ordinary giant magnon and single spike of the undeformed background. The solutions live in an $S^{3}$ subspace of the five-sphere in which the $B$-field has non-zero components. Furthermore, the solutions are not point like but extend in the Schrödinger part of the metric. ${ }^{1}$

In [23] three-point correlation functions involving two heavy operators and a light one were calculated using holography in a Schrödinger background. These are the first results in the literature of three-point function computations involving extended string solutions. In [24] the pp-wave geometry of the Schrödinger background was constructed. The spectrum of the bosonic excitations was derived and compelling agreement with the giant magnon dispersion relation, previously obtained in [20], was found. In [25] the giant graviton solution of the Schrödinger pp-wave geometry [24] was constructed. The solution exhibits an intriguing behavior as the deformation parameter varies, which is suggestive of a spontaneous breaking of conformal invariance. Finally, pulsating strings solutions in the Schrödinger background were constructed in [26].

The aim of this work is to continue in the direction of further studying the gauge/gravity correspondence, in order to relate the non-supersymmetric Schrödinger background to its dual null dipole-deformed $\mathcal{N}=4 \mathrm{SYM}$ [27-29]. To that extent, in this paper we will further elaborate on the classical string solutions that were constructed in [20]. We will calculate the finite size (exponential) corrections to the infinite size dispersion relation of the single spin giant magnon and of the single spin single spike solutions. The solutions live in a $S^{3}$ subspace of the five-sphere, extent in the Schrödinger part of the metric and in the limit of zero deformation the finite size dispersion relations flow to the undeformed $\operatorname{AdS}_{5} \times S^{5}$ counterparts [30, 31].

The paper is organized as follows: in section 2 we review the single spin giant magnon and the single spin single spike solutions in the Schrodinger background. The ansatz for the solutions was introduced in [20,23] but the boundary conditions are revised in order to accommodate the finite size corrections. In section 3 we focus on the giant magnon case and we calculate the explicit expressions for the conserved charges. Afterwards, we expand the dispersion relation around the infinite size limit and the zero deformation limit. In section 4 we focus on the single spike case and after calculating the explicit expressions for the conserved charges we expand the dispersion relation around the infinite size limit. We conclude the paper in section 5. In appendices $\mathrm{A}$ and $\mathrm{B}$ we present details of the computations of the main text.

\section{Classical string solutions on $\mathrm{Sch}_{5} \times S^{3}$}

In this section we will review the single spin giant magnon and the single spin single spike solutions in the Schrodinger $\operatorname{Sch}_{5} \times S^{5}$ background, that were initially presented in [20, 23].

\footnotetext{
${ }^{1}$ Giant-magnon like solutions with a different dispersion relation were studied in [21], while giant magnons and spiky strings living on the Schrödinger $\operatorname{Sch}_{5} \times T^{1,1}$ and the corresponding dispersion relations were studied in [22].
} 
More specifically, in [23] the dispersion relations for the infinite size single spin giant magnon and the infinite size single spin single spike were presented, and it is those relations that in the next section we will generalize to include the finite size corrections.

We consider the following consistent truncation of the 10d Schrodinger $\operatorname{Sch}_{5} \times S^{5}$ background on a sphere ${ }^{2}$

$$
d s^{2}=-\left(1+\frac{\mu^{2}}{Z^{4}}\right) d T^{2}+\frac{1}{Z^{2}}\left(2 d T d V+d Z^{2}\right)+d \eta^{2}+\sin ^{2} \eta d \varphi_{1}^{2}+\cos ^{2} \eta d \varphi_{2}^{2}
$$

that is supplemented with a $B$-field

$$
B=\frac{\mu}{Z^{2}} d T \wedge\left(\sin ^{2} \eta d \varphi_{1}+\cos ^{2} \eta d \varphi_{2}\right)
$$

where $\eta \in[0, \pi / 2], \varphi_{1} \in[0,2 \pi)$ and $\varphi_{2} \in[0,2 \pi)$ are the ranges of the variables along $S^{3}$.

\subsection{Ansatz and equations of motion}

We consider the following ansatz for both solutions

$$
\begin{aligned}
& T=\kappa \tau, \quad V=\alpha \tau+V_{y}(y), \quad Z=Z_{0}, \quad \eta=\frac{1}{2} \theta_{y}(y), \\
& \varphi_{1}=\omega \tau+\frac{\kappa \mu}{Z_{0}^{2}} \sigma+\Psi_{y}(y) \quad \& \quad \varphi_{2}=-\frac{\kappa \mu}{Z_{0}^{2}} \sigma
\end{aligned}
$$

where we have defined the variable $y$ as

$$
y \equiv c \sigma-d \tau .
$$

The explicit expressions for the functions $V_{y}(y), \theta_{y}(y)$ and $\Psi_{y}(y)$ that appear in the ansatz will be determined through the equations of motion and the Virasoro constraints, while $\kappa$, $\alpha, Z_{0}, \omega$ and $\mu$ are constants.

Notice that even if we are considering single spin solutions, the string needs to move inside an $S^{3}$, rather than an $S^{2}$, subspace of the 5 -sphere. In order to have a dispersion relation that depends on the deformation, we are forced to switch on a third angle inside the 5 -sphere. This is not something unusual when we study the motion of classical strings inside deformations of the AdS space (e.g. see the case of the giant magnon inside the beta-deformed $\mathrm{AdS}_{5} \times S^{5}$ background [32]).

The equations for $V_{y}^{\prime}(y)$ and $\Psi_{y}^{\prime}(y)$ in terms of the new function $u(y),{ }^{3}$ are given in appendix A, while the equation of motion for $Z$ and one of the Virasoro constraints give us the following two conditions

$$
A_{T} d Z_{0}^{2}-2 A_{\phi} c \mu+\alpha c^{2}-d^{2} \kappa Z_{0}^{2}=0
$$

and

$$
\frac{A_{\phi} \omega}{d}-\frac{A_{T} \kappa}{2 d}+\frac{1}{Z_{0}^{2}}\left(\frac{\alpha \kappa}{2}+\frac{A_{\phi} \kappa \mu}{c}\right)=0 .
$$

\footnotetext{
${ }^{2}$ With respect to the notation of [20] for the $S^{3}$, we have performed a change of variables: $\theta=2 \eta$, $\psi=\varphi_{1}+\varphi_{2}$ and $\phi=\varphi_{1}-\varphi_{2}$. Details about the consistency of the truncation can be found in [20].

${ }^{3}$ The functions $\theta_{y}(y)$ and $u(y)$ are related as follows: $u \equiv \cos ^{2}\left(\frac{\theta_{y}}{2}\right)$.
} 
The equation of motion for $u(y)$ is coming from the other Virasoro constraint and it is

$$
\frac{\left(u^{\prime}\right)^{2}}{2}+\mathcal{W}(u)=0 \quad \text { with } \quad \mathcal{W}(u)=-2 u\left(\beta_{6} u^{2}+\beta_{4} u+\beta_{2}\right)
$$

where the constants $\beta_{2}, \beta_{4} \& \beta_{6}$ are listed in appendix $\mathrm{A}$. It is possible to rewrite equation (2.7) in the following way

$$
u^{\prime}=2 \sqrt{\left|\beta_{6}\right| u\left(u_{p}-u\right)\left(u-u_{m}\right)} \quad \text { with } \quad u_{m}<u<u_{p}
$$

where

$$
u_{p}=\frac{1}{2}\left[-\frac{\beta_{4}}{\beta_{6}}+\sqrt{\left(\frac{\beta_{4}}{\beta_{6}}\right)^{2}-4 \frac{\beta_{2}}{\beta_{6}}}\right] \quad \& \quad u_{m}=-\frac{1}{2}\left[\frac{\beta_{4}}{\beta_{6}}+\sqrt{\left(\frac{\beta_{4}}{\beta_{6}}\right)^{2}-4 \frac{\beta_{2}}{\beta_{6}}}\right] .
$$

In the infinite size limit, where $\beta_{6}<0, \beta_{4}>0$ and $\beta_{2}=0$, the bending point of the string is at $u_{m}=0$.

\subsection{Boundary conditions}

In this subsection we will impose boundary conditions to the equations of motion of the previous subsection, in analogy to the infinite size case of [20].

Solving (2.5) with respect to $A_{T}$ we obtain the following expression

$$
A_{T}=\frac{1}{d Z_{0}^{2}}\left(2 A_{\phi} c \mu-\alpha c^{2}+d^{2} \kappa Z_{0}^{2}\right) .
$$

Substituting (2.10) in (A.1) and imposing that $V_{y}^{\prime}$ vanishes at the bending point $u=u_{m}$ we obtain the following two solutions for the constant $A_{\phi}$

$$
\begin{array}{ll}
A_{\phi}=\frac{\alpha}{2 c \kappa \mu^{2}}\left[c^{2} \kappa \mu+c d \omega Z_{0}^{2}+d^{2} \kappa \mu\right] & \text { single spin giant magnon } \\
A_{\phi}=\frac{c \alpha}{2 \mu} & \text { single spin single spike. }
\end{array}
$$

Substituting the expression for $A_{T}$ and $A_{\phi}$ (from (2.10) and (2.11) respectively) in (2.9), we express $u_{m}$ (of the giant magnon) in terms of the auxiliary quantity $W_{\mathrm{GM}}$, as follows

$$
u_{m}=1-W_{\mathrm{GM}} \quad \text { with } \quad W_{\mathrm{GM}}=\frac{\alpha Z_{0}^{2}}{\kappa \mu^{2}} .
$$

The expression for $u_{p}$ (of the giant magnon) becomes

$$
u_{p}=1-v^{2} W_{\mathrm{GM}}\left[1+\frac{1-v^{2}}{v} \frac{\Delta_{\mathrm{GM}}}{W_{\mathrm{GM}}+2 v \Delta_{\mathrm{GM}}}\right]^{2} \quad \text { with } \quad \Delta_{\mathrm{GM}}=\frac{\alpha}{\mu \omega} \quad \& \quad v=\frac{d}{c} .
$$

The expressions for $Z_{0}$ and $\kappa$ in terms of the auxiliary quantities $W_{\mathrm{GM}}$ and $\Delta_{\mathrm{GM}}$ are

$$
Z_{0}=\sqrt{\frac{\kappa}{\alpha}} \mu \sqrt{W_{\mathrm{GM}}} \quad \& \quad \kappa=\omega\left[W_{\mathrm{GM}}+2 v \Delta_{\mathrm{GM}}+\left(1+v^{2}\right) \frac{\Delta_{\mathrm{GM}}^{2}}{W_{\mathrm{GM}}}\right]^{1 / 2} .
$$


Notice, that the undeformed limit of the expressions above is for $\Delta_{\mathrm{GM}} \rightarrow 0$ (or equivalently $\alpha \rightarrow 0$ ) and the infinite size limit is for $W_{\mathrm{GM}} \rightarrow 1$.

A similar analysis can be performed also for the single spin single spike case. Now the starting point for the value of the constant $A_{\phi}$ will be (2.12). The expression for $u_{m}$ (of the single spike) in terms of the auxiliary quantity $W_{\mathrm{SS}}$ becomes

$$
u_{m}=1-W_{\mathrm{SS}} \quad \text { with } \quad W_{\mathrm{SS}}=\frac{\alpha}{\mu v}\left(\omega+\frac{2 \kappa \mu}{v Z_{0}^{2}}\right)^{-1} .
$$

The expression for $u_{p}$ (of the single spike) becomes

$$
u_{p}=1-v^{2} W_{\mathrm{SS}} \quad \text { with } \quad v=\frac{c}{d}
$$

and for later convenience we introduce the following auxiliary quantity $\Delta_{\mathrm{SS}}$

$$
\Delta_{\mathrm{SS}}=\frac{\mu \omega}{\alpha} .
$$

The expressions for $Z_{0}$ and $\kappa$ in terms of the auxiliary quantities $W_{\mathrm{SS}}$ and $\Delta_{\mathrm{SS}}$ are

$$
Z_{0}=\frac{\sqrt{2 \kappa \alpha W_{\mathrm{SS}}} \Delta_{\mathrm{SS}}}{\omega \sqrt{1-v W_{\mathrm{SS}} \Delta}} \quad \& \quad \kappa=\frac{\omega}{\sqrt{W_{\mathrm{SS}}} \Delta_{\mathrm{SS}}} .
$$

Notice, that the undeformed limit of the expressions above is for $\Delta_{\mathrm{SS}} \rightarrow \frac{1}{v W_{\mathrm{SS}}}$ (or equivalently $\left.Z_{0} \rightarrow \infty\right)$ and the infinite size limit is for $W_{\mathrm{SS}} \rightarrow 1$.

\section{Single spin giant magnon}

In this section we compute the conserved charges for the single spin giant magnon solution and construct the dispersion relation. The detailed analysis for the calculation of the dispersion relation in the infinite size limit appears in [20,23]. In this section we focus on the finite size corrections of the aforementioned relation.

The four conserved charges, that originate from the partial derivatives of the Polyakov action and the subsequent integration, for the giant magnon solution become ${ }^{4}$

$$
\begin{aligned}
\frac{E}{2 T} & =\frac{1-v^{2}}{\sqrt{u_{p}}} \frac{W}{W+2 v \Delta}\left[W+2 v \Delta+\left(1+v^{2}\right) \frac{\Delta^{2}}{W}\right]^{1 / 2} \mathbf{K}(1-\epsilon) \\
\frac{\mu M}{2 T} & =\frac{1-v^{2}}{\sqrt{u_{p}}} \frac{\Delta}{W+2 v \Delta} \mathbf{K}(1-\epsilon) \\
\frac{J}{2 T} & =\frac{1}{\sqrt{u_{p}}}\left[1-\frac{v^{2} W}{W+2 v \Delta}\left(W+\frac{1+v^{2}}{v} \Delta\right)\right] \mathbf{K}(1-\epsilon)-\sqrt{u_{p}} \mathbf{E}(1-\epsilon) \\
\frac{\Delta \varphi}{2} & =\frac{v W+\left(1+v^{2}\right) \Delta}{(W+2 v \Delta) \sqrt{u_{p}}}\left[\frac{W}{1-u_{p}} \mathbf{\Pi}\left(-\frac{u_{p}}{1-u_{p}}(1-\epsilon), 1-\epsilon\right)-\mathbf{K}(1-\epsilon)\right]
\end{aligned}
$$

\footnotetext{
${ }^{4}$ In order for the notation not to clutter, in this section $W$ is $W_{\mathrm{GM}}$ from (2.13) and $\Delta$ is $\Delta_{\mathrm{GM}}$ from (2.14).
} 
where $\epsilon$ is the ratio between $u_{m}$ and $u_{p}$ of the giant magnon solution

$$
\epsilon=\frac{u_{m}}{u_{p}}
$$

and $\mathbf{K}(1-\epsilon), \mathbf{E}(1-\epsilon)$ and $\mathbf{\Pi}\left(-\frac{u_{p}}{1-u_{p}}(1-\epsilon), 1-\epsilon\right)$ are the complete elliptic integrals of the first, the second and the third kind. In appendix $\mathrm{B}$ we have gathered all the expressions that define those integrals. The finite size corrections we will calculate are in powers of the ratio $\epsilon$ when this ratio is small. For this reason we expand the parameters $v$ and $W$ in powers of $\epsilon$ as follows

$$
v=v_{0}+\left(v_{1}+v_{2} \log \epsilon\right) \epsilon \quad \& \quad W=W_{0}+W_{1} \epsilon
$$

and the presence of the logarithmic term is for the compensation of the logarithmic terms that come from the expansion of the elliptic integrals. Using the definition for $\epsilon$ from (3.5) and the condition $\Delta \varphi=p$, it is possible to determine all the coefficients of the above expansion. The coefficients of the $W$ expansion are simple expressions and do not depend on the deformation parameter $\Delta$

$$
W_{0}=1 \quad \& \quad W_{1}=-\sin ^{2} \frac{p}{2} .
$$

On the contrary, the coefficients of the $v$ expansion depend on the deformation parameter and they have analytic but non illuminating expressions. We list them in appendix B and more specifically in equations (B.4), (B.5) and (B.6). Here we present the expansion of the coefficients around the undeformed values, namely for small values of $\Delta$. For the coefficient $v_{0}$ the expansion becomes

$$
\frac{v_{0}}{\cos \frac{p}{2}}=1-\frac{\sin ^{2} \frac{p}{2}}{\cos \frac{p}{2}} \Delta+\mathcal{O}\left(\Delta^{3}\right)
$$

for the coefficient $v_{1}$ it is

$$
\frac{v_{1}}{\frac{1}{4} \sin ^{2} \frac{p}{2} \cos \frac{p}{2}(1-4 \ln 2)}=1+\frac{2-6 \sin ^{2} \frac{p}{2}-8 \cos ^{2} \frac{p}{2} \ln 2}{\cos \frac{p}{2}(1-4 \ln 2)} \Delta+\mathcal{O}\left(\Delta^{3}\right)
$$

and for the coefficient $v_{2}$ it is

$$
\frac{v_{2}}{\frac{1}{4} \sin ^{2} \frac{p}{2} \cos \frac{p}{2}}=1+2 \cos \frac{p}{2} \Delta+\mathcal{O}\left(\Delta^{3}\right) .
$$

Notice here, that the current calculation of finite size corrections in the dispersion relation of the giant magnon only makes sense in the limit of small $\Delta$. Increasing the value of the deformation parameter, increases the ratio between $u_{m}$ and $u_{p}$ and as a result cancels the expansion in small $\epsilon$. The expansion is justified only for small values of $\Delta$.

From the zeroth order term in the expansion of $J$, we obtain the expression of $\epsilon$ for $J \gg T$

$$
\epsilon=16 \exp \left[\left[-\frac{J}{T \sin \frac{p}{2}}-2\right]\left[1-\Delta \cos \frac{p}{2}\right]\right]
$$

where we have expanded the exponent in powers of $\Delta$. 
Using all those ingredients it is possible to expand the dispersion relation of the giant magnon. The zeroth order term provides the infinite size result while the first order correction depends on the value of $\Delta$. For small values of $\Delta$ the dispersion relation is

$$
\begin{aligned}
\frac{\sqrt{E^{2}-\mu^{2} M^{2}}-J}{2 T \sin \frac{p}{2}}-1= & -4 \sin ^{2} \frac{p}{2} e^{-\frac{J}{T \sin \frac{p}{2}}-2} \\
& +2 \Delta^{2}\left[\frac{J^{2}}{T^{2}} \cos ^{2} \frac{p}{2}+2 \frac{J}{T} \cos p \sin \frac{p}{2}-4 \sin ^{4} \frac{p}{2}\right] e^{-\frac{J}{T \sin \frac{p}{2}}-2}
\end{aligned}
$$

For $\Delta=0$ we obtain the undeformed result of [30] (see also [31]) and in the infinite size limit the r.h.s. of the finite size dispersion relation vanishes.

\section{Single spin single spike}

In this section, we compute the conserved charges for the single spin single spike solution and construct the dispersion relation. The detailed analysis for the calculation of the dispersion relation in the infinite size limit appears in $[20,23]$. In this section we focus on the finite size corrections of the aforementioned relation.

The four conserved charges for the single spike solution become ${ }^{5}$

$$
\begin{aligned}
\frac{E}{2 T} & =\sqrt{\frac{1}{v^{2}}-1} \sqrt{1-\epsilon} \mathbf{K}(1-\epsilon) \\
\frac{\mu M}{2 T} & =\frac{1}{2}(1-v W \Delta) \sqrt{\frac{1}{v^{2}}-1} \sqrt{1-v^{2} \epsilon} \mathbf{K}(1-\epsilon) \\
\frac{J}{2 T} & =\sqrt{\frac{1-v^{2}}{1-v^{2} \epsilon}}[\mathbf{E}(1-\epsilon)-\epsilon \mathbf{K}(1-\epsilon)] \\
\frac{\Delta \varphi}{2} & =\sqrt{\frac{1-v^{2} \epsilon}{1-v^{2}}}\left[\left[v+\frac{1-v^{2}}{2 v}(1-v W \Delta)\right] \mathbf{K}(1-\epsilon)-\frac{1}{v} \mathbf{\Pi}\left(1-\frac{1}{v^{2}}, 1-\epsilon\right)\right]
\end{aligned}
$$

where the constant $W$ can be expressed as a function of $\epsilon$ as follows

$$
W=\frac{1-\epsilon}{1-v^{2} \epsilon}
$$

and $\epsilon$ is again the ratio between $u_{m}$ and $u_{p}$ (of the single spike). The next step is to expand $v$ in powers of $\epsilon$ (see equation (3.6)), substitute it in the expression for $J / T$ from (4.3) and impose that $J / T$ remains finite. In that way, we determine the value of the coefficients $v_{0}$, $v_{1}$ and $v_{2}$ in terms of the finite quantity $J / T$

$$
v_{0}^{2}=1-\frac{1}{4} \frac{J^{2}}{T^{2}}, \quad v_{1}=-\frac{1-v_{0}^{2}}{4 v_{0}}\left[1+4 \ln 2-2 v_{0}^{2}\right] \quad \& \quad v_{2}=\frac{1-v_{0}^{2}}{4 v_{0}} .
$$

\footnotetext{
${ }^{5}$ In this section $W$ is $W_{\mathrm{SS}}$ from $(2.16)$ and $\Delta$ is $\Delta_{\mathrm{SS}}$ from (2.18).
} 
From the $\epsilon$-expansion of $\Delta \varphi$, we obtain the expression for $\epsilon$ as a function of $\Delta \varphi$ and $J / T$

$$
\epsilon=16 \exp \left[-\frac{4 \sqrt{4-\mathcal{J}^{2}}}{\mathcal{J}} \frac{\Delta \varphi+\arcsin \left(\frac{\mathcal{J}}{2} \sqrt{4-\mathcal{J}^{2}}\right)}{2+\Delta \sqrt{4-\mathcal{J}^{2}}}\right] \quad \text { with } \quad \mathcal{J}=\frac{J}{T} .
$$

Using all those ingredients it is possible to expand the dispersion relation of the single spike. The zeroth order term provides the infinite size result while the first order correction depends on the value of $\Delta$. Here contrary to the giant magnon result the expressions are simple and we do not expand in powers of the deformation. The finite size dispersion relation is

$$
\frac{1}{2 T}[E-\mu M-\Delta \varphi]-\frac{p}{2}=4 \sin ^{2} \frac{p}{2} \tan \frac{p}{2} \exp \left[-\frac{\Delta \varphi+p}{\tan \frac{p}{2}} \frac{2}{1+\Delta \cos \frac{p}{2}}\right]
$$

where we have used the identification that was introduced in [17]

$$
\arcsin \left(\frac{J}{2 T}\right)=\frac{p}{2}
$$

The undeformed result of [31] is realized for $\Delta=\cos ^{-1} \frac{p}{2}$ (this is the same value of $\Delta$ for which $\left.Z_{0} \rightarrow \infty\right)$ and in the infinite size limit the r.h.s. of the finite size dispersion relation vanishes.

\section{Conclusions}

In this paper we have elaborated on the dispersion relations of semi-classical string solutions that live in the Schrödinger spacetime, that is conjectured to be the gravity dual of the null dipole CFT. We have calculated the finite size corrections to the infinite size dispersion relation of the single spin giant magnon and of the single spin single spike solutions. The leading order corrections have the usual exponential form, characteristic for the finite size corrections also in the original $\mathrm{AdS}_{5} \times S^{5}$ background, dressed with expressions that depend on the deformation parameter. In the limit of zero deformation, the solutions and their finite size dispersion relations become those of the single spin giant magnon and single spin single spike of the undeformed $\mathrm{AdS}_{5} \times S^{5}$ background. Furthermore, in the infinite size limit the correction term vanishes and we obtain the known infinite size dispersion relations. Even if the solutions presented in this paper are single spin they live in an $S^{3}$ subspace of the five-sphere. In order to have a dispersion relation that depends on the deformation, we are forced to let the string move inside an $S^{3}$, instead of the $S^{2}$ that the string would move in the undeformed counterpart.

A number of important directions remains to be addressed. In the current paper we have calculated the finite size corrections for the single spin giant magnon and single spike. It would be interesting to generalize the computation for the dyonic giant magnon and single spike. The string solutions and the corresponding infinite size dispersion relations have been studied in [20]. In a very recent interesting approach, the classical exponential corrections to the dispersion relations of the GKP string, of the giant magnon and of the 
single spike have been expressed in terms of Lambert W-function [33-35]. In this way the leading, sub-leading and next-to-sub-leading series of the classical exponential corrections to the dispersion relations of the aforementioned string configurations have been calculated. It would be very interesting to follow this path in the Schrödinger geometry in order to expand the computation of the current paper beyond the leading order.

\section{Acknowledgments}

We would like to thank George Georgiou for reading very carefully the preliminary draft and sending various useful comments. We are grateful to Georgios Linardopoulos for collaboration in the early stages of this work. The work of this project has received funding from the Hellenic Foundation for Research and Innovation (HFRI) and the General Secretariat for Research and Technology (GSRT), under grant agreement No. 15425.

\section{A Useful expressions from the analysis of the classical string solutions}

In this appendix we gather all the useful (but lengthy and not particularly illuminating) expressions from the detailed analysis of the classical string solutions, that is presented in section 2 .

The derivative of the function $V_{y}(y)$ with respect to $y$ in terms of $u(y)$ is given by

$$
V_{y}^{\prime}(y)=\frac{1}{c^{2}-d^{2}}\left[\left(d \kappa-A_{T}\right) Z_{0}^{2}-\alpha d+c \mu \omega\left(1+\frac{2 d \kappa \mu}{c \omega Z_{0}^{2}}\right)(1-u(y))\right]
$$

while the derivative of the function $\Psi_{y}(y)$ with respect to $y$ is given by

$$
\Psi_{y}^{\prime}(y)=\frac{1}{c^{2}-d^{2}}\left[\frac{2 A_{\phi}}{1-u(y)}-d \omega-\frac{2 c \kappa \mu}{Z_{0}^{2}}\right] .
$$

The constants $\beta_{2}, \beta_{4} \& \beta_{6}$ from the equation of motion for the function $u(y)$ in (2.7) are given by the following expressions

$$
\begin{aligned}
\beta_{4}= & \frac{1}{c d Z_{0}^{2}\left(c^{2}-d^{2}\right)^{2}}\left[-2 A_{\phi}\left(c Z_{0}^{2} \omega+d \kappa \mu\right)\left(c^{2}+d^{2}\right)-4 A_{\phi} c^{2} d \kappa \mu\right. \\
& \left.+c \kappa\left(c^{2}-d^{2}\right)\left[Z_{0}^{2}\left(A_{T}-d \kappa\right)+\alpha d\right]+\frac{2 c d}{Z_{0}^{2}}\left(c Z_{0}^{2} \omega+2 d \kappa \mu\right)^{2}\right] \\
\beta_{6}= & -\frac{c^{2} \omega^{2}}{\left(c^{2}-d^{2}\right)^{2}}\left(1+\frac{2 d \kappa \mu}{c \omega Z_{0}^{2}}\right)<0 \quad \& \quad \beta_{2}+\beta_{4}+\beta_{6}=-\frac{4 A_{\phi}^{2}}{\left(c^{2}-d^{2}\right)^{2}} .
\end{aligned}
$$

\section{B Elliptic integrals and $\epsilon$ expansion coefficients of the parameter $v$}

In this appendix we gather all the useful expressions for the definitions of the elliptic integrals (of the first, second and third type) that we used to express the conserved quantities, 
both for the giant magnon and single spike solutions. These are

$$
\begin{aligned}
\int_{u_{m}}^{u_{p}} \frac{d u}{\sqrt{u\left(u_{p}-u\right)\left(u-u_{m}\right)}} & =\frac{2}{\sqrt{u_{p}}} \mathbf{K}(1-\epsilon) \\
\int_{u_{m}}^{u_{p}} \frac{\sqrt{u} d u}{\sqrt{\left(u_{p}-u\right)\left(u-u_{m}\right)}} & =2 \sqrt{u_{p}} \mathbf{E}(1-\epsilon) \\
\int_{u_{m}}^{u_{p}} \frac{d u}{(1-u) \sqrt{u\left(u_{p}-u\right)\left(u-u_{m}\right)}} & =\frac{2}{\left(1-u_{p}\right) \sqrt{u_{p}}} \mathbf{\Pi}\left(\frac{u_{m}-u_{p}}{1-u_{p}} \mid 1-\epsilon\right) .
\end{aligned}
$$

The expressions for the $\epsilon$ expansion coefficients of the parameter $v$ in the single spin giant magnon solution of section 3 are

$$
\begin{aligned}
& v_{0}=\frac{1}{2 \Delta}\left[\sqrt{1+4 \Delta\left[\Delta \cos p+\cos \frac{p}{2} \sqrt{1-4 \Delta^{2} \sin ^{2} \frac{p}{2}}\right]}-1\right] \\
& v_{2}=\frac{\left(1-v_{0}^{2}\right)\left[v_{0}+\left(1+v_{0}^{2}\right) \Delta\right]}{4\left(1+2 v_{0} \Delta\right)}\left[1+\frac{\left(1-v_{0}^{2}\right) \Delta^{2}}{1+2 \Delta\left[v_{0}-\Delta\left(1-v_{0}^{2}\right)\right]}\right] \\
& \frac{v_{1}}{v_{2}}=\frac{v_{0}(1-4 \ln 2)\left[1+2 \Delta^{2}\left(1+v_{0}^{2}\right)\right]-\Delta(3+4 \ln 2)+v_{0}^{2} \Delta(7-12 \ln 2)}{\left(1+2 v_{0} \Delta\right)\left[v_{0}+\left(1+v_{0}^{2}\right) \Delta\right]} .
\end{aligned}
$$

Open Access. This article is distributed under the terms of the Creative Commons Attribution License (CC-BY 4.0), which permits any use, distribution and reproduction in any medium, provided the original author(s) and source are credited.

\section{References}

[1] N. Beisert et al., Review of AdS/CFT Integrability: An Overview, Lett. Math. Phys. 99 (2012) 3 [arXiv: 1012.3982] [INSPIRE].

[2] J.M. Maldacena, The Large $N$ limit of superconformal field theories and supergravity, Int. J. Theor. Phys. 38 (1999) 1113 [Adv. Theor. Math. Phys. 2 (1998) 231] [hep-th/9711200] [INSPIRE].

[3] A.V. Ramallo, Introduction to the AdS/CFT correspondence, Springer Proc. Phys. 161 (2015) 411 [arXiv: 1310.4319] [INSPIRE].

[4] J.D. Edelstein, J.P. Shock and D. Zoakos, The AdS/CFT Correspondence and Non-perturbative QCD, AIP Conf. Proc. 1116 (2009) 265 [arXiv:0901.2534] [InSPIRE].

[5] J.A. Minahan and K. Zarembo, The Bethe ansatz for $N=4$ superYang-Mills, JHEP 03 (2003) 013 [hep-th/0212208] [INSPIRE].

[6] K. Zarembo, Holographic three-point functions of semiclassical states, JHEP 09 (2010) 030 [arXiv: 1008.1059] [INSPIRE].

[7] M.S. Costa, R. Monteiro, J.E. Santos and D. Zoakos, On three-point correlation functions in the gauge/gravity duality, JHEP 11 (2010) 141 [arXiv:1008.1070] [INSPIRE].

[8] G. Georgiou, Two and three-point correlators of operators dual to folded string solutions at strong coupling, JHEP 02 (2011) 046 [arXiv: 1011.5181] [INSPIRE]. 
[9] G. Georgiou, SL(2) sector: weak/strong coupling agreement of three-point correlators, JHEP 09 (2011) 132 [arXiv: 1107.1850] [INSPIRE].

[10] J. Escobedo, N. Gromov, A. Sever and P. Vieira, Tailoring Three-Point Functions and Integrability, JHEP 09 (2011) 028 [arXiv: 1012.2475] [INSPIRE].

[11] Y. Kazama, S. Komatsu and T. Nishimura, Classical Integrability for Three-point Functions: Cognate Structure at Weak and Strong Couplings, JHEP 10 (2016) 042 [Erratum JHEP 02 (2018) 047] [arXiv: 1603.03164] [INSPIRE].

[12] D.M. Hofman and J.M. Maldacena, Giant Magnons, J. Phys. A 39 (2006) 13095 [hep-th/0604135] [INSPIRE].

[13] J.A. Minahan, A. Tirziu and A.A. Tseytlin, Infinite spin limit of semiclassical string states, JHEP 08 (2006) 049 [hep-th/0606145] [INSPIRE].

[14] H.-Y. Chen, N. Dorey and K. Okamura, Dyonic giant magnons, JHEP 09 (2006) 024 [hep-th/0605155] [INSPIRE].

[15] S. Benvenuti and E. Tonni, Giant magnons and spiky strings on the conifold, JHEP 02 (2009) 041 [arXiv: 0811.0145] [INSPIRE].

[16] M. Kruczenski, J. Russo and A.A. Tseytlin, Spiky strings and giant magnons on $S^{5}$, JHEP 10 (2006) 002 [hep-th/0607044] [inSPIRE].

[17] R. Ishizeki and M. Kruczenski, Single spike solutions for strings on $S^{2}$ and $S^{3}$, Phys. Rev. D 76 (2007) 126006 [arXiv: 0705.2429] [INSPIRE].

[18] M. Guica, F. Levkovich-Maslyuk and K. Zarembo, Integrability in dipole-deformed $\mathcal{N}=4$ super Yang-Mills, J. Phys. A 50 (2017) 394001 [arXiv:1706. 07957] [INSPIRE].

[19] H. Ouyang, Semiclassical spectrum for BMN string in $\mathrm{Sch}_{5} \times S^{5}$, JHEP 12 (2017) 126 [arXiv: 1709.06844] [INSPIRE].

[20] G. Georgiou and D. Zoakos, Giant magnons and spiky strings in the Schrödinger/dipole-deformed CFT correspondence, JHEP 02 (2018) 173 [arXiv:1712.03091] [INSPIRE].

[21] C. Ahn and P. Bozhilov, Giant magnon-like solution in $\mathrm{Sch}_{5} \times S^{5}$, Phys. Rev. D 98 (2018) 106005 [arXiv: 1711.09252] [INSPIRE].

[22] A. Golubtsova, H. Dimov, I. Iliev, M. Radomirov, R.C. Rashkov and T. Vetsov, More on Schrödinger holography, arXiv:2004.13802 [INSPIRE].

[23] G. Georgiou and D. Zoakos, Holographic three-point correlators in the Schrödinger/dipole CFT correspondence, JHEP 09 (2018) 026 [arXiv: 1806.08181] [INSPIRE].

[24] G. Georgiou, K. Sfetsos and D. Zoakos, String theory on the Schrödinger pp-wave background, JHEP 08 (2019) 093 [arXiv:1906. 08269] [INSPIRE].

[25] G. Georgiou and D. Zoakos, Giant gravitons on the Schrödinger pp-wave geometry, JHEP 03 (2020) 185 [arXiv : 2002.05460] [INSPIRE].

[26] H. Dimov, M. Radomirov, R.C. Rashkov and T. Vetsov, On pulsating strings in Schrödinger backgrounds, JHEP 10 (2019) 094 [arXiv: 1903. 07444] [INSPIRE].

[27] J.M. Maldacena, D. Martelli and Y. Tachikawa, Comments on string theory backgrounds with non-relativistic conformal symmetry, JHEP 10 (2008) 072 [arXiv:0807.1100] [INSPIRE]. 
[28] C.P. Herzog, M. Rangamani and S.F. Ross, Heating up Galilean holography, JHEP 11 (2008) 080 [arXiv: 0807.1099] [INSPIRE].

[29] A. Adams, K. Balasubramanian and J. McGreevy, Hot Spacetimes for Cold Atoms, JHEP 11 (2008) 059 [arXiv: 0807.1111] [INSPIRE].

[30] G. Arutyunov, S. Frolov and M. Zamaklar, Finite-size Effects from Giant Magnons, Nucl. Phys. B 778 (2007) 1 [hep-th/0606126] [InSPIRE].

[31] C. Ahn and P. Bozhilov, Finite-size Effects for Single Spike, JHEP 07 (2008) 105 [arXiv: 0806.1085] [INSPIRE].

[32] C.-S. Chu, G. Georgiou and V.V. Khoze, Magnons, classical strings and $\beta$-deformations, JHEP 11 (2006) 093 [hep-th/0606220] [INSPIRE].

[33] G. Georgiou and G. Savvidy, Large spin behavior of anomalous dimensions and short-long strings duality, J. Phys. A 44 (2011) 305402 [arXiv: 1012.5580] [INSPIRE].

[34] E. Floratos, G. Georgiou and G. Linardopoulos, Large-Spin Expansions of GKP Strings, JHEP 03 (2014) 018 [arXiv: 1311.5800] [INSPIRE].

[35] E. Floratos and G. Linardopoulos, Large-Spin and Large-Winding Expansions of Giant Magnons and Single Spikes, Nucl. Phys. B 897 (2015) 229 [arXiv:1406.0796] [INSPIRE]. 